\title{
Mass as a Geometric Property of Spacetime
}

\author{
Kazuo Ota Cottrell ${ }^{1,2}$ \\ I(Physics Department, University of Massachusetts Dartmouth, United States of America) \\ ${ }^{2}$ (Present Address: 1107 Lind Street, Middletown OH 45044 USA)
}

\begin{abstract}
A relationship between mass as a geometric concept and motion associated with a closed curve in spacetime (a notion taken from differential geometry) is investigated. We show that the 4-dimensional exterior Schwarzschild solution of the General Theory of Relativity can be mapped to a 4-dimensional Euclidean spacetime manifold. As a consequence of this mapping, the quantity $M$ in the exterior Schwarzschild solution which is usually attributed to a massive central object is shown to correspond to a geometric property of spacetime. An additional outcome of this analysis is the discovery that, because $M$ is a property of spacetime geometry, an anisotropy with respect to its spacetime components measured in a Minkowski tangent space defined with respect to a spacetime event $P$ by an observer $O$ who is stationary with respect to the spacetime event $P$, may be a sensitive measure of an anisotropic cosmic accelerated expansion. The presence of anisotropy in the cosmic accelerated expansion may contribute to the reason that there are currently two prevailing measured estimates of this quantity.
\end{abstract}

Keywords: cosmic accelerated expansion, Euclidean spacetime, gravitational and inertial mass, Schwarzschild solution, Weitzenböck manifold

\section{Introduction}

In 1919, an eclipse[1] expedition organized by Sir Arthur Eddington provided what was considered at the time to be conclusive evidence for the correctness of the General Theory of Relativity by procuring a measurement of the deflection angle of light grazing the solar limb which matched the prediction of Einstein's theory within a $20 \%$ margin of error. In recent times, the development of Yang-Mills gravity has created a need for reconsideration of measurements of the deflection of starlight by the Sun as a means of resolving the difference in the predictions made for this phenomenon by the two theories.[2][3] Unfortunately, current measurements in the optical region of the spectrum lack adequate precision. The upcoming total solar eclipse of the Sun on August 21, 2017 will provide an outstanding opportunity to obtain new, more precise measurements.

The purpose of this paper is not just to encourage an improvement in the precision of measurements of the total deflection angle of starlight by the Sun, but to motivate a new approach to looking at the problem which involves treating mass as a geometric quantity. It is the belief of the author that such a new perspective will be required to resolve the question of the relative merits of Yang-Mills gravity versus Einstein's General Theory of Relativity.

In section two, we develop a generalization of the idea of four-vector force from the flat-spacetime of the Special Theory of Relativity to an expression which allows one to compare components of the four-vector force at different locations in a Riemann-Cartan spacetime that possesses zero curvature, i.e., a Weitzenböck manifold. In the third section of this paper, we introduce the idea of inertial mass as a geometric quantity. In section four we complete our demonstration that the generalized four-vector developed in section two corresponds to a geometric quantity. In section five, we argue that a spherical gravitational mass M may be represented by the spacetime components of proper inertial mass corresponding to a coordinate system having its origin defined at the center of the spherical mass. In section six, we apply this result and show that it is possible to map the 4-dimensional geometry of the exterior Schwarzschild solution to a 4-dimensional Euclidean spacetime manifold. In the seventh section, we continue our analysis by further developing its consequences: in particular, we complete our journey by presenting the analogue for Newton's second law, expressed in a spacetime having zero curvature, but nonzero torsion. In section eight, we summarize our conclusions.

\section{The Four Vector Force and the Nature of Inertial Mass}

The usefulness of vectors as a description of physical quantities is largely due to the remarkable property of vectors in Euclidean spacetime that they remain invariant with respect to translation. Thus, a vector provides the same description of a physical quantity no matter what coordinate system is used for the description of a particular physical phenomenon which constitutes a spacetime event, as long as spacetime is Euclidean. In this sense, a vector behaves as a simpler version of a more general mathematical object known as a tensor. A 
tensor is more general because it can describe physical quantities that are characterized by more than one direction in a multidimensional spacetime.

In a Riemann-Cartan spacetime, however, vectors no longer possess this simple property. When a vector is translated along a closed curve in a Riemann-Cartan spacetime, one observes that the vector has undergone two changes: a rotation, or reorientation of the direction of the vector in the Riemann-Cartan spacetime, and a translation of the point which marks the tip of the vector in the tangent space defined at the origin of the vector in the Riemann-Cartan spacetime.

The first type of change, i.e., the reorientation of the vector in Riemann-Cartan spacetime, may be attributed to a property known as curvature. One may compensate for the effect of curvature on the translation of a vector by introducing a quantity known as a Levi-Civita connection (often referred to as Christoffel symbols). Here, however, we consider the simpler special case of a Riemann-Cartan spacetime manifold for which the curvature vanishes, i.e., for which:

$$
\mathrm{R}_{i j k}^{l}=0
$$

where $\mathrm{R}^{1}{ }_{\mathrm{ijk}}$ is the Riemann curvature tensor. In this special case of a Riemann-Cartan spacetime, a vector is not rotated upon being translated along a closed curve. Such a spacetime is said to be described by a Weitzenböck manifold.

There still remains to be considered, however, the second type of change, i.e., the translation of the tip of the vector in the tangent space defined at the origin of the vector in the Weitzenböck spacetime. In a paper written in 1977, Hayashi and Shirafuji[4] astutely pointed out this type of change, which they called "the concept of motion associated with a closed curve in an affinely connected space-time manifold", and suggested that for gravity the concept of the motion associated with a loop is much more important than that of a nonintegrable phase factor.

This assertion was confirmed by the work of Bennett, Das, Laperashvili, and Nielson[5] when they showed, in work done in 2012, that in the formulation of four-dimensional gravity by Plebanski, gravitation described in terms of a Riemann-Cartan spacetime without curvature, but possessing torsion (i.e., a Weitzenböck manifold) is equivalent to the usual model of gravitation developed by Einstein in his General Theory of Relativity, expressed in terms of a curved Riemannian manifold.

In this paper, we attempt to extend the applicability of the concept of motion associated with a closed curve to the general concept of force as defined by a four-vector translated along a closed curve in a Weitzenböck manifold, to obtain a broader basis for unification of gravitation with the other gauge forces.

Newton's second law, expressed within the context of a spacetime with zero curvature, but nonzero torsion may be written as:

$$
\mathrm{F}^{a b}=m^{a} \mathrm{a}^{b}
$$

The superscript indices a and $\mathrm{b}$ take on the values 0 through 3 . When taken to correspond to tetrad indices, analogous to methods employed by Einstein when considering distant parallelism[6] these indices are necessary to acknowledge the fact that Newton's second law, written in its usual form as the following 3-vector equation:

$$
\mathbf{F}=m \mathbf{a}
$$

is strictly valid only when the Riemann curvature tensor is zero:

$$
\mathrm{R}_{i j k}^{l}=0
$$

In this paper, however, lower case Latin indices $(a, b, \ldots)$ denote the directions corresponding to the differential elements of coordinate length which define the spacetime translation of the point which defines the tip of the corresponding vector in a tangent Minkowski space when a force vector is translated along a closed curve in the Weitzenböck manifold. Ozakin and Yavari have demonstrated that such a spacetime translation corresponds to the displacement defined by a mathematical object corresponding to the Burgers vector of crystallography defined for the continuum case, where the density of this continuum version of the Burgers vector is given by a torsion tensor that corresponds to the density of dislocations in the elastic medium.[7]

Equation (2.3) tells us that the acceleration of mass is an expression of force. Similarly, from the perspective of (2.2) one may write:

$$
?=\frac{d x^{\alpha}}{c_{0}} \mathrm{a}^{b}
$$

and ask, "the acceleration of spacetime is an expression of what quantity?" Considering unit analysis, it makes sense to let:

$$
?=\mathrm{V}^{\alpha b}
$$

where $\mathrm{V}^{\alpha b}$ is an unknown quantity having the units of velocity. It now follows from (2.5) and (2.6) that: 


$$
\mathrm{V}^{\alpha b}=\frac{d x^{\alpha}}{c_{0}} a^{b} \Rightarrow g_{\alpha \beta} \frac{d x^{\beta}}{c_{0}} \mathrm{~V}^{\alpha b}=\frac{g_{\alpha \beta} d x^{\alpha} d x^{\beta}}{c_{0}{ }^{2}} \mathrm{a}^{b}
$$

or:

$$
\mathrm{a}^{b}=\frac{\mathrm{V}_{\beta}^{b} d x^{\beta}}{c_{0} d \tau^{2}}
$$

where we have used the fact that:

$$
g_{\alpha \beta} d x^{\alpha} d x^{\beta}=c_{0}^{2} d \tau^{2}
$$

Substituting (2.8) into (2.2) one arrives at:

$$
\mathrm{F}^{a b}=\frac{m^{a} \mathrm{~V}_{\beta}^{b} d x^{\beta}}{c_{0} d \tau^{2}}
$$

Let us now consider the following quantity:

$$
\left(\frac{\mathrm{V}_{\beta}^{b} d x^{\beta}}{c_{0} d \tau^{2}}\right)
$$

Note that the following correspondence exists:

$$
\mathrm{U}^{\beta}=\frac{d x^{\beta}}{d \tau} \Leftrightarrow \mathrm{U}_{b}=\frac{d x_{b}}{d \tau}
$$

where $\mathrm{U}^{\beta}$ is the usual four-vector velocity defined for flat spacetime, and $\mathrm{U}_{b}$ is the corresponding one-form in a Minkowski cotangent space. Making this correspondence, one may define the following quantity:

$$
\mathrm{a}_{\beta} \equiv\left(\frac{\mathrm{V}_{\beta}^{b} \mathrm{U}_{b}}{c_{0} d \tau}\right)
$$

This allows us to write (2.10) in the following way:

$$
\mathrm{F}_{\beta}^{a}=m^{a} \mathrm{a}_{\beta}
$$

Equation (2.14) allows one to compare components of force at different locations of a Riemann-Cartan spacetime which has zero curvature, i.e., one for which (2.1) holds true. Equation (2.14) may be rearranged to provide an expression for $m^{a}$ :

$$
m^{a}=\frac{\mathrm{F}_{\beta}^{a}}{\mathrm{a}_{\beta}}
$$

In this sense, inertial mass is now seen to be a geometric property of spacetime geometry.

\section{Inertial Mass as a Geometric Quantity}

Let us now see how our new understanding of the role of inertial mass as a geometric property of spacetime allows us to redefine the increments of length associated with the spacetime coordinate system of observer $\mathrm{O}$, who is stationary with respect to spacetime event $\mathrm{P}$, which marks the origin of the four-vector force $\mathrm{F}^{a}{ }_{\beta}$, as a set of dyads of the form:

$$
\left\{\left(d x^{0},\left(m_{O}\right)^{0}\right),\left(d x^{1},\left(m_{O}\right)^{1}\right),\left(d x^{2},\left(m_{O}\right)^{2}\right),\left(d x^{3},\left(m_{O}\right)^{3}\right)\right\}
$$

From the perspective of (2.14), force is now a kinematic, rather than a dynamic quantity. In other words, it is a property which describes the state of motion of a spacetime event without reference to any external agency or cause. Likewise, momentum, too, is a kinematic quantity, and the expression for the four-momentum is given by:

$$
\mathrm{P}_{\alpha}^{a}=\left(m_{O}\right)^{a} \mathrm{U}_{\alpha}
$$

The subscript $\mathrm{O}$ is used to specify the fact that $\left(m_{O}\right)^{a}$ here is a property of a spacetime coordinate system which is at rest with respect to observer $\mathrm{O}$. The relationship between the four-vector force and the four-vector momentum is given by:[8] 


$$
\mathrm{F}_{\beta}^{a}=\frac{d \mathrm{P}_{\beta}^{a}}{d t}=\left(m_{O}\right)^{a} \frac{d \mathrm{U}_{\beta}}{d t}
$$

Combining (2.14) with (3.3) yields:

$$
m^{a} \mathrm{a}_{\beta}=\left(m_{O}\right)^{a} \frac{d \mathrm{U}_{\beta}}{d t}
$$

which may be rearranged to give:

$$
\left(m_{O}\right)^{a}=\frac{m^{a} \mathrm{a}_{\beta} d t}{d \mathrm{U}_{\beta}}
$$

Equation (3.5) relates the inertial mass measured by observer $O$ to correspond to spacetime translational component "a" in the Minkowski tangent space at his or her particular location in spacetime (i.e., the proper inertial mass associated with spacetime component " $\mathrm{a}$ " at a particular spacetime event $\mathrm{P}$ ) to the inertial mass measured for spacetime translational component "a" at that same spacetime event $\mathrm{P}$ by an arbitrary observer moving with four-vector acceleration $\mathrm{a}_{\beta}$ relative to the event at $\mathrm{P}$.

Strictly speaking, to validate our assertion that $\left(m_{O}\right)^{a}$ is truly a geometric quantity, we must show that the quantity $m^{a} \mathrm{a}_{\beta}$ (i.e., $\mathrm{F}_{\beta}^{a}$ ) appearing on the right hand side of (3.5) depends only on $\left(m_{O}\right)^{a}$ and other kinematic quantities. We proceed to do this in the next section.

\section{Four Vector Force in Terms of Proper Inertial Mass}

We now wish to express the four-vector acceleration defined by (2.13) and the four-vector force defined by (2.14), respectively, in a way that makes explicit their dependence on the proper inertial mass $m_{O}$ and its spacetime components $\left(m_{O}\right)^{a}$, without reference to the inertial mass $m^{a}$ of an arbitrary accelerating observer. Rearranging (2.13), we obtain:

$$
\mathrm{V}_{\beta}^{b}=\frac{\mathrm{a}_{\beta} d \tau \mathrm{U}^{b}}{c_{0}}
$$

where we have used the fact that:

$$
\mathrm{U}^{b} \mathrm{U}_{b}=c_{0}^{2}
$$

Substituting (2.13) into (3.5) one obtains:

$$
\left(m_{O}\right)^{a}=\frac{m^{a} \mathrm{~V}_{\beta}^{b} \mathrm{U}_{b} d t}{c_{0} d \mathrm{U}_{\beta} d \tau}
$$

Now, by making the following replacements in (4.3):

we obtain:

$$
\mathrm{U}_{b} \rightarrow \frac{d \omega}{d k^{b}}, d k^{b} \rightarrow \frac{d p^{b}}{\hbar}
$$

$$
\left(m_{O}\right)^{a}=\frac{\hbar d \omega m^{a} \mathrm{~V}_{\beta}^{b} d t}{c_{0} d \mathrm{p}^{b} d \mathrm{U}_{\beta} d \tau}
$$

Next, using (4.1) to replace $\mathrm{V}_{\beta}{ }^{b}$ in (4.5) allows us to reintroduce $\mathrm{a}_{\beta}$ into this expression:

$$
\left(m_{O}\right)^{a}=\frac{\hbar d \omega \mathrm{m}^{a} \mathrm{a}_{\beta} \mathrm{U}^{b} d t}{c_{0}{ }^{2} d \mathrm{p}^{b} d \mathrm{U}_{\beta}}
$$

Next, making the replacements: 


$$
\begin{aligned}
& \hbar \mathrm{d} \omega d t=(h /(2 \pi))(2 \pi d v) d t \rightarrow h \\
& d \mathrm{p}^{b} \rightarrow \gamma d\left(\mathrm{p}_{N}\right)^{b}, d\left(p_{N}\right)^{b}=\gamma^{-1} m_{O} d \mathrm{U}^{b} \\
& \gamma=\frac{1}{\sqrt{1-\frac{c^{2}}{c_{0}^{2}}}}, \mathrm{c}^{2}=\mathrm{u}^{b} \mathrm{u}_{b}, \mathrm{u}^{b}=\gamma^{-1} \mathrm{U}^{b} \\
& \therefore \gamma=\sqrt{2} \\
& d \mathrm{U}_{\beta} \rightarrow \gamma d \mathrm{u}_{\beta}
\end{aligned}
$$

we obtain:

$$
\gamma\left(m_{O}\right)^{a}=m^{a}=\frac{h \mathrm{~m}^{a} \mathrm{a}_{\beta} \gamma \mathrm{u}^{b}}{m_{O} c_{0}{ }^{2} d \mathrm{U}^{b} d \mathrm{u}_{\beta}}
$$

Solving the second equality in (4.8) for the four-vector acceleration, $\mathrm{a}_{\beta}$, we obtain:

$$
\mathrm{a}_{\beta}=\frac{c_{0}^{2} d\left(\mathrm{p}_{N}\right)^{b} d \mathrm{u}_{\beta}}{h \mathrm{u}^{b}}
$$

which simplifies to:

$$
\mathrm{a}_{\beta}=\frac{m_{O} c_{0}^{2} d \mathrm{u}^{b} d \mathrm{u}_{\beta}}{h \mathrm{u}^{b}}
$$

where we have used the fact (from (4.7)) that:

$$
d\left(\mathrm{p}_{N}\right)^{b}=m_{O} d \mathrm{u}^{b}
$$

One may rewrite (4.10) in the following way:

$$
\mathrm{a}_{\beta}=\lambda_{C}{ }^{-1} c_{0}\left(\frac{d c_{0}}{c_{0}}+\frac{d \mathrm{u}^{1}}{\mathrm{u}^{1}}+\frac{d \mathrm{u}^{2}}{\mathrm{u}^{2}}+\frac{d \mathrm{u}^{3}}{\mathrm{u}^{3}}\right) d \mathrm{u}_{\beta}
$$

where we have introduced the definition of the Compton wavelength:

$$
\lambda_{C}=\frac{h}{m_{O} c_{0}}
$$

Since $\mathrm{c}_{0}$ is a constant, $\mathrm{dc}_{0}=0$, so the first term in parenthesis in (4.12) vanishes, and we are left with:

$$
\mathrm{a}_{\beta}=\lambda_{C}{ }^{-1} c_{0}\left(\frac{d \mathrm{u}^{1}}{\mathrm{u}^{1}}+\frac{d \mathrm{u}^{2}}{\mathrm{u}^{2}}+\frac{d \mathrm{u}^{3}}{\mathrm{u}^{3}}\right) d \mathrm{u}_{\beta}
$$

The four-vector acceleration $a_{\beta}$ is by nature a kinematic quantity, as it can be used to specify a description of motion without regard to a causative (i.e., external physical) source. In other words, it is due to a property of spacetime. This assertion is consistent with Einstein's geometrical description of gravitation, which thus does not require explanation of motion in terms of dynamical interactions. From this perspective, (4.10) may be rearranged to express $\mathrm{m}_{\mathrm{O}}$ as a geometric quantity:

$$
m_{O}=\frac{h \mathrm{a}_{\beta}}{c_{0}^{2}\left(\frac{d \mathrm{u}^{1}}{\mathrm{u}^{1}}+\frac{d \mathrm{u}^{2}}{\mathrm{u}^{2}}+\frac{d \mathrm{u}^{3}}{\mathrm{u}^{3}}\right) d \mathrm{u}_{\beta}}
$$

Substituting (4.14) into (2.15) we obtain the corresponding definition of $m^{a}$ :

$$
m^{a}=\frac{\mathrm{F}_{\beta}^{a}}{\lambda_{C}{ }^{-1} c_{0}\left(\frac{d \mathrm{u}^{1}}{\mathrm{u}^{1}}+\frac{d \mathrm{u}^{2}}{\mathrm{u}^{2}}+\frac{d \mathrm{u}^{3}}{\mathrm{u}^{3}}\right) d \mathrm{u}_{\beta}}
$$

Using (4.16), one can make more explicit the idea of inertial mass as a kinematic (i.e., geometric), rather than a dynamic (i.e., causative) quantity. With the help of (3.3), one may rewrite (4.16) as: 


$$
m^{a}=\frac{\left(m_{O}\right)^{a} d \mathrm{U}_{\beta}}{\lambda_{C}{ }^{-1} c_{0}\left(\frac{d \mathrm{u}^{1}}{\mathrm{u}^{1}}+\frac{d \mathrm{u}^{2}}{\mathrm{u}^{2}}+\frac{d \mathrm{u}^{3}}{\mathrm{u}^{3}}\right) d \mathrm{u}_{\beta} d t}
$$

which relates the inertial mass measured to correspond to spacetime translational component " $\mathrm{a}$ " in the Minkowski tangent space for a four-vector force $\mathrm{F}_{\beta}^{a}$ which has moved along a closed curve in spacetime, returning to its origin at spacetime event $\mathrm{P}$, by an arbitrary observer $O^{\prime}$ who is moving with four-vector acceleration relative to the event at $\mathrm{P}$, to the proper inertial mass measured to correspond to spacetime translational component "a" in the Minkowski tangent space defined at $\mathrm{P}$, by an observer $O$ who remains stationary with respect to spacetime event $P$.

Finally, using (2.15) to replace $m^{a}$ on the left hand side of (4.17) one obtains (with help from (4.7)) the desired expression for the four-vector force in terms of the proper inertial mass $\mathrm{m}_{\mathrm{O}}$ and its geometric components $\left(m_{O}\right)^{a}:$

$$
\mathrm{F}_{\beta}^{a}=\frac{\sqrt{2}\left(m_{O}\right)^{a} \mathrm{a}_{\beta}}{\lambda_{C}{ }^{-1} c_{0}\left(\frac{d \mathrm{u}^{1}}{\mathrm{u}^{1}}+\frac{d \mathrm{u}^{2}}{\mathrm{u}^{2}}+\frac{d \mathrm{u}^{3}}{\mathrm{u}^{3}}\right) d t}
$$

Equation (4.18) allows one to connect the idea of four-vector force as a dymanic quantity found in the flat spacetime of Einstein's Special Theory of Relativity, to a generalized representation as a four-vector defined by (3.3) in a Riemann-Cartan spacetime having zero curvature, but nonzero torsion (i.e., a Weitzenböck manifold), allowing one to develop an idea of force as a geometric property of spacetime, consistent with the view of Einstein's General Theory of Relativity. However, (4.18) is of general applicability, not being restricted to forces of a gravitational nature. Therefore, for example, since $\mathrm{F}_{\beta}^{a}$ which appears on the left hand side of (4.18) may represent a force which is electromagnetic in nature, (4.18) provides a generalization of the equivalence principle which allows one to describe forces of an arbitrary nature in terms of kinematic quantities which are described in terms of the geometry of a Riemann-Cartan spacetime.

\section{Introduction of the Gravitational Mass M}

If the representation of four-vector force in a Weitzenböck manifold, $\mathrm{F}_{\beta}^{a}$, given in (4.18) and $\mathrm{u}^{\mathrm{i}}$ are independent of time, we may perform separation of variables and integrate (4.18):

$$
\left(\sqrt{2} \lambda_{C}\right)^{-1} c_{0} \int \mathrm{F}_{\beta}^{a} \sum_{i=1}^{3} d\left(\ln \left[\mathrm{u}^{i}\right]\right)=\int\left(m_{O}\right)^{a} \mathrm{a}_{\beta} d v
$$

If $\left(m_{O}\right)^{a}$ and $\mathrm{a}_{\beta}$ are independent of time, we may thus express (4.18) in the following way:

$$
\int \mathrm{F}_{\beta}^{a} \sum_{i=1}^{3} d\left(\ln \left[\mathrm{u}^{i}\right]\right) \equiv \mathrm{F}_{\text {Geometric }}=\frac{\left(m_{O}\right)^{a} \mathrm{a}_{\beta} \sqrt{2} \lambda_{C} \nu}{c_{0}}
$$

By using (3.3) to substitute for $\mathrm{F}_{\beta}^{a}$ on the left hand side of (5.2) and using the definition:

$$
\mathrm{a}_{\beta}=\frac{d \mathrm{U}_{\beta}}{d t}
$$

which is valid for constant $\mathrm{a}_{\beta}$ we then obtain the following result:

$$
v=\left(\sqrt{2} \lambda_{C}\right)^{-1} c_{0} \sum_{i=1}^{3} \ln \left[\mathrm{u}^{i}\right]
$$

which defines a frequency associated with motion in a three-dimensional Euclidean space. This frequency corresponds to the de Broglie frequency, which, expressed in isotropic coordinates $\rho$, is given by:

$$
v_{\text {de Broglie }}=\frac{m_{0} c_{0}^{2}}{\sqrt{2} h(\mathrm{n}[\rho])^{2}}
$$

provided that the following is true: 


$$
\mathrm{n}[\boldsymbol{\rho}]=\left(\sum_{i=1}^{3} \ln \left[\mathrm{u}^{i}\right]\right)^{-\frac{1}{2}}
$$

Moshinsky[9] has shown that from an electromagnetic viewpoint, the gravitational field of a star makes the surrounding space behave like an inhomogeneous material medium with relative permittivity $\varepsilon_{\mathrm{r}}$ and relative permeability $\mu_{\mathrm{r}}$ given by:

$$
\varepsilon_{\mathrm{r}}=1+\frac{2 G M}{c_{0}^{2} \rho}, \mu_{\mathrm{r}}=\left(1-\frac{2 G M}{c_{0}^{2} \rho}\right)^{-1}
$$

Then the effective index of refraction in the space around the star may be expressed as:

$$
\mathrm{n}[\rho]=\frac{c_{0}}{c}=\frac{(\varepsilon \mu)^{\frac{1}{2}}}{\left(\varepsilon_{0} \mu_{0}\right)^{\frac{1}{2}}}=\left(\varepsilon_{\mathrm{r}} \mu_{\mathrm{r}}\right)^{\frac{1}{2}}
$$

Substituting (5.7) into (5.8) one obtains:

$$
\mathrm{n}[\rho]=\frac{\left(1+\frac{2 G M}{c_{o}^{2} \rho}\right)^{\frac{1}{2}}}{\left(1-\frac{2 G M}{c_{o}^{2} \rho}\right)^{\frac{1}{2}}}
$$

Combining (5.6) and (5.9), one finds the following relation:

$$
\left(\sum_{i=1}^{3} \ln \left[\mathrm{u}^{i}\right]\right)=\frac{\left(1-\frac{2 G M}{c_{o}^{2} \rho}\right)}{\left(1+\frac{2 G M}{c_{o}^{2} \rho}\right)}
$$

Since the left hand side of (5.10) contains only kinematic quantities, it is evident that the right hand side represents a geometric quantity. If we now consider the observer $O$ to be defined by a coordinate system having its origin at spacetime event $\mathrm{P}$ located at the center of spherical mass $\mathrm{M}$, with $\mathrm{P}$ not located at the origin of the coordinate system of an arbitrary observer $O^{\prime}$ (we set this restriction, because although the field point corresponding to the measurement of a physical quantity by an arbitrary observer may take on any value, a source point may not be located at the origin of the coordinate system for this observer) who is moving with respect to spacetime event $\mathrm{P}$ with four-vector acceleration $\mathrm{a}_{\beta}$, then the experimentally demonstrated equivalence between gravitational and inertial mass allows us to reexpress (5.9) geometrically, in the following way:

$$
\mathrm{n}[\rho]=\left(\sum_{i=1}^{3} \ln \left[\mathrm{u}^{i}\right]\right)^{-\frac{1}{2}}=\frac{\left(1+\frac{2 G\left(m_{O}\right)^{a} \hat{\mathrm{e}}_{a}}{c_{o}^{2} \rho}\right)^{\frac{1}{2}}}{\left(1-\frac{2 G\left(m_{O}\right)^{a} \hat{\mathrm{e}}_{a}}{c_{o}^{2} \rho}\right)^{\frac{1}{2}}}
$$

\section{Euclidean Form for the Static, Isotropic Schwarzschild Metric}

The conception of mass as a geometric quantity is not new. For example, this idea becomes evident from a consideration of the Schwarzschild solution of the vacuum form of Einstein's field equations.[10] In this section, we wish to consider the most general Euclidean line element corresponding to a static, isotropic metric, which takes the form:

$$
d s^{2}=\mathrm{A}(\rho) c_{0}{ }^{2} d t^{2}+\mathrm{B}(\rho) d \sigma^{2}
$$

and show that the Schwarzschild line element expressed in isotropic coordinates may be written in this form. 
In Riemann-Cartan spacetime without curvature, but with nonzero torsion (where torsion may be thought of as a form of kinematic curvature, or curvature associated with the spacetime coordinate system), or equivalently in the curved Riemannian manifold of Einstein's General Theory of Relativity, in the presence of free-fall acceleration, one has the following relationships:

$$
c d t=d \sigma
$$

and:

$$
c d \tau=d s
$$

Squaring (6.2) and (6.3) and then subtracting the former from the latter we find:

$$
c^{2} d \tau^{2}-c^{2} d t^{2}=d s^{2}-d \sigma^{2}
$$

One may pass from the description in Riemann-Cartan and Riemannian spacetimes, respectively, to a Euclidean spacetime described by spherical coordinates, by introducing the following line element:

$$
d s^{2}=c^{2} d t^{2}+d \sigma^{2}
$$

where:

$$
d \sigma^{2}=d \rho^{2}+d \rho^{2} d \theta^{2}+d \rho^{2} \sin ^{2} \theta d \varphi^{2}
$$

or:

$$
d \sigma^{2}=d \rho^{2}\left(1+d \theta^{2}+\sin ^{2} \theta d \varphi^{2}\right)
$$

To obtain the form of (6.7) it has been necessary to apply the following transformation to the last two terms (i.e., the terms with angular dependence) of the usual form of the line element for spatial spherical coordinates:

$$
\rho^{2} \rightarrow d \rho^{2}
$$

because although Euclidean spacetime is flat in the sense of zero curvature (i.e., (2.1) applies), it is expressed within the context of this paper as a Riemann-Cartan spacetime with zero curvature, in the limit as the torsion approaches zero, not by setting the torsion identically equal to zero.

Using (6.5) to replace $\mathrm{ds}^{2}$ on the right hand side of (6.4) and rearranging, one obtains:

$$
c^{2} d \tau^{2}=2 c^{2} d t^{2}
$$

Substituting (6.9) into the left hand side of (6.4) and rearranging, we recover:

$$
d s^{2}=c^{2} d t^{2}+d \sigma^{2}
$$

which verifies that (6.2), (6.3), (6.4), and (6.5) are mutually consistent.

The most general Euclidean line element thus has the form:

$$
d s^{2}=\mathrm{A}^{\prime}(\rho) c^{2} d t^{2}+\mathrm{B}^{\prime}(\rho) d \sigma^{2}
$$

or:

$$
d s^{2}=\frac{\mathrm{A}^{\prime}(\rho)}{\mathrm{n}[\rho]^{2}} c_{0}{ }^{2} d t^{2}+\mathrm{B}^{\prime}(\rho) d \sigma^{2}
$$

The most general form for the Schwarzschild line element in isotropic spherical coordinates is:

$$
d s^{2}=\mathrm{C}(\rho) c_{0}^{2} d t^{2}-\mathrm{D}(\rho) d \sigma_{S}^{2}
$$

The coefficients $C(\rho)$ and $D(\rho)$ may be computed, following the method employed by Adler, Bazin, and Schiffer:[11]

$$
\mathrm{C}(\rho)=\frac{\left(1-\frac{G M}{2 c_{0}^{2} \rho}\right)^{2}}{\left(1+\frac{G M}{2 c_{0}^{2} \rho}\right)^{2}}, \mathrm{D}(\rho)=\left(1+\frac{G M}{2 c_{0}^{2} \rho}\right)^{4}
$$

so that the Schwarzschild line element in isotropic spherical coordinates is:

$$
d s^{2}=\frac{\left(1-\frac{G M}{2 c_{0}^{2} \rho}\right)^{2}}{\left(1+\frac{G M}{2 c_{0}^{2} \rho}\right)^{2}} c_{0}^{2} d t^{2}-\left(1+\frac{G M}{2 c_{0}^{2} \rho}\right)^{4} d \sigma_{S}^{2}
$$


When the interval is light-like, $\mathrm{ds}^{2}=0$, and the line elements of (6.12) and (6.15) may be set equal, allowing us to compare the coefficients. Before comparing coefficients, however, one must note that $\mathrm{d} \sigma^{2}$ for the Euclidean line element, as given by (6.7) differs from $\mathrm{d} \sigma_{\mathrm{S}}{ }^{2}$ for the Schwarzschild line element, which is given by:

Rearranging (6.16) we find:

$$
d \sigma_{S}^{2}=d \rho^{2}+\rho^{2} d \theta^{2}+\rho^{2} \sin ^{2} \theta d \varphi^{2}
$$

$$
d \rho^{2}=d \sigma_{S}^{2}-\rho^{2} d \theta^{2}-\rho^{2} \sin ^{2} \theta d \varphi^{2}
$$

Substituting (6.17) into (6.7) one obtains:

$$
d \sigma^{2}=\left[d \sigma_{S}^{2}-\rho^{2}\left(d \theta^{2}+\sin ^{2} \theta d \varphi^{2}\right)\right]\left(1+d \theta^{2}+\sin ^{2} \theta d \varphi^{2}\right)
$$

which may be rearranged to yield:

$$
d \sigma_{S}^{2}=\frac{d \sigma^{2}+\rho^{2}\left(d \theta^{2}+\sin ^{2} \theta d \varphi^{2}\right)\left(1+d \theta^{2}+\sin ^{2} \theta d \varphi^{2}\right)}{\left(1+d \theta^{2}+\sin ^{2} \theta d \varphi^{2}\right)}
$$

Thus, by substitution of (6.19) into (6.15), one obtains the following form for the Schwarzschild line element:

$$
d s^{2}=\frac{\left(1-\frac{G M}{2 c_{0}^{2} \rho}\right)^{2}}{\left(1+\frac{G M}{2 c_{0}{ }^{2} \rho}\right)^{2}} c_{0}^{2} d t^{2}-\left(1+\frac{G M}{2 c_{0}^{2} \rho}\right)^{4} d \rho\left[1+\left(d \theta^{2}+\sin ^{2} \theta d \varphi^{2}\right)\right]
$$

where we have used the fact that:

$$
\frac{d \sigma^{2}}{d \rho^{2}}=\left(1+d \theta^{2} \sin ^{2} \theta d \varphi^{2}\right)
$$

and applied the required transformation (6.8) to the line element. Thus, by considering the case when the interval is light-like, and comparing (6.12) to (6.20) we find:

$$
\mathrm{A}^{\prime}(\rho)=\mathrm{C}(\rho) \mathrm{n}[\rho]^{2}=\frac{\left(1-\frac{G\left(m_{O}\right)^{a} \hat{\mathrm{e}}_{a}}{2 c_{0}^{2} \rho}\right)^{2}}{\left(1+\frac{G\left(m_{O}\right)^{a} \hat{\mathrm{e}}_{a}}{2 c_{0}^{2} \rho}\right)^{2}} \frac{\left(1+\frac{2 G\left(m_{O}\right)^{a} \hat{\mathrm{e}}_{a}}{c_{o}^{2} \rho}\right)}{\left(1-\frac{2 G\left(m_{O}\right)^{a} \hat{\mathrm{e}}_{a}}{c_{o}^{2} \rho}\right)}
$$

and:

$$
\mathrm{B}^{\prime}(\rho)=i^{2} \mathrm{D}(\rho)=i^{2}\left(1+\frac{G\left(m_{o}\right)^{a} \hat{\mathrm{e}}_{a}}{2 c_{0}^{2} \rho}\right)^{4}
$$

where we have used (5.11) and the corresponding geometric representation of (6.14). So, the Schwarzschild line element expressed in isotropic spherical coordinates may be expressed as a line element for a Euclidean spacetime in the following way:

$$
d s^{2}=\frac{\left(1-\frac{G\left(m_{O}\right)^{a} \hat{\mathrm{e}}_{a}}{2 c_{0}{ }^{2} \rho}\right)^{2}}{\left(1+\frac{G\left(m_{O}\right)^{a} \hat{\mathrm{e}}_{a}}{2 c_{0}{ }^{2} \rho}\right)^{2}} c_{0}{ }^{2} d t^{2}+\left(1+\frac{G\left(m_{O}\right)^{a} \hat{\mathrm{e}}_{a}}{2 c_{0}{ }^{2} \rho}\right)^{4}(i d \sigma)^{2}
$$

Next, we note that:

$$
d \mathrm{u}^{a}=\frac{\partial \mathrm{u}^{a}}{\partial \sigma} d \sigma+\frac{\partial \mathrm{u}^{a}}{\partial t} d t
$$

Taking $\mathrm{u}^{a}$ to be independent of time at the location of spacetime event $\mathrm{P}$ so that: 


$$
\left.\frac{\partial \mathrm{u}^{a}}{\partial t}\right|_{P}=0
$$

we find that the last term of (6.25) vanishes. Now, applying the definition:

$$
d \mathrm{u}^{a}=\nabla \mathrm{u}^{a} d \sigma
$$

we are able to conclude that:

$$
\nabla \mathrm{u}^{a}=\frac{\partial \mathrm{u}^{a}}{\partial \sigma}
$$

This allows us to express d $\sigma$ in the following way:

$$
d \sigma=\frac{d \mathrm{u}^{a}}{\nabla \mathrm{u}^{a}}
$$

Additionally, we may express $\mathrm{dt}$ in the following way:

$$
d t=\frac{d u^{a}}{\mathrm{a}^{a}}
$$

Introducing (6.29) and (6.30) into (6.24), we may express the Euclidean form of the line element corresponding to the Schwarzschild solution of the vacuum form of the Einstein field equations as:

$$
d s^{2}=\frac{\left(1-\frac{G\left(m_{O}\right)^{a} \hat{\mathrm{e}}_{a}}{2 c_{0}^{2} \rho}\right)^{2}}{\left(1+\frac{G\left(m_{O}\right)^{a} \hat{\mathrm{e}}_{a}}{2 c_{0}^{2} \rho}\right)^{2}} \frac{c_{0}{ }^{2}\left(d \mathrm{u}^{a}\right)^{2}}{\left(\mathrm{a}^{a}\right)^{2}}+\left(1+\frac{G\left(m_{O}\right)^{a} \hat{\mathrm{e}}_{a}}{2 c_{0}{ }^{2} \rho}\right)^{4}\left(\frac{i}{\nabla \mathrm{u}^{a}}\right)^{2}\left(d \mathrm{u}^{a}\right)^{2}
$$

In concluding this section, we note that many preliminary efforts have been made toward the direction of achieving a quantum theory of gravity. These efforts have achieved partial success, but have not yet led to a decisive final theory. Interestingly, many of these attempts have started from the viewpoint of a relativistic framework for quantum mechanics, i.e., the framework of quantum field theory. Yet, in his seminal paper which united the foundations of quantum electrodynamics, Freeman Dyson[12] pointed out that relativistic quantum mechanics is a special case of non-relativistic quantum mechanics. Thus, it makes sense that a framework with the potential for providing a broader base for attempts to unify gravitation with the other gauge forces should include ideas fundamental to non-relativistic quantum mechanics, but not necessarily the full machinery required for quantum field theory. Taking this viewpoint, and noting that the momentum operator for nonrelativistic quantum mechanics is given by:[13]

$$
\hat{\mathbf{p}}=-i \hbar \nabla
$$

we rewrite (6.31), without loss of generality, with the introduction of the four vector version, $\hat{\mathbf{P}}$, of the nonrelativistic quantum mechanical momentum operator in the following way:

$$
d s^{2}=\left[\frac{\left(1-\frac{G\left(m_{O}\right)^{a} \hat{\mathrm{e}}_{a}}{2 c_{0}{ }^{2} \rho}\right)^{2}}{\left(1+\frac{G\left(m_{O}\right)^{a} \hat{\mathrm{e}}_{a}}{2 c_{0}{ }^{2} \rho}\right)^{2}} \frac{c_{0}{ }^{2}}{\left(\mathrm{a}^{a}\right)^{2}}+\left(1+\frac{G\left(m_{O}\right)^{a} \hat{\mathrm{e}}_{a}}{2 c_{0}{ }^{2} \rho}\right)^{4}\left(\frac{\hbar}{\hat{\mathbf{P}}^{a}}\right)^{2}\right]\left(d \mathrm{u}^{a}\right)^{2}
$$

Equation (6.33) has been developed in this paper in a way which is consistent with the principles of the Special and General Theories of Relativity. The fact that the quantum mechanical momentum operator appears in the Euclidean form of the line element corresponding to the Schwarzschild solution of the vacuum form of the Einstein field equations in this way suggests the intriguing possibility that the generalization of the equivalence principle discussed with regard to gravitation and forces of an electromagnetic nature when considering (4.18) may be extended to a description of forces in the quantum domain, allowing the additional inclusion of gauge forces having only a microscopic range. From the viewpoint of non-relativistic quantum mechanics, this would entail replacing the classical form of Newton's second law, given by (2.3) with the use of Ehrenfest's theorem:[13] 


$$
\frac{d}{d t}\langle\mathbf{p}\rangle=-\int \psi *(\nabla V) \psi d \rho^{3} \sin \theta d \theta d \varphi=-\langle V\rangle=\langle\mathbf{F}\rangle
$$

where $V$ here is the scalar potential corresponding to the force $\mathbf{F}$, and \langle\rangle indicates the expectation value of the quantity contained inside. Note also that transformation (6.8) has been applied when writing the volume element, so that the resulting integral is five dimensional.

\section{Consequences of the Geometric Nature of Mass for our Physical Description of Nature}

We now wish to consider the consequences of the formalism developed in this paper for our description of Nature. One modification introduced by applying the formalism developed in this paper, which follows from the representation of the gravitational mass, $\mathrm{M}$, as a geometric quantity, is the replacement of the Schwarzschild radius, $\mathrm{r}_{\mathrm{s}}$, with the following geometric expression:

$$
\mathrm{R}_{\mathrm{S}} \equiv\left(\mathrm{R}_{\mathrm{S}}\right)^{a} \hat{\mathrm{e}}_{a}=\frac{2 G\left(m_{O}\right)^{a} \hat{\mathrm{e}}_{a}}{c_{0}{ }^{2}}
$$

In other words, we write (6.33) as:

Noting that:

$$
d s^{2}=\left[\frac{\left(1-\frac{\mathrm{R}_{\mathrm{S}}}{4 \rho}\right)^{2}}{\left(1+\frac{\mathrm{R}_{\mathrm{S}}}{4 \rho}\right)^{2}} \frac{c_{0}{ }^{2}}{\left(\mathrm{a}^{a}\right)^{2}}+\left(1+\frac{\mathrm{R}_{\mathrm{S}}}{4 \rho}\right)^{4}\left(\frac{\hbar}{\hat{\mathbf{P}}^{a}}\right)^{2}\right]\left(d \mathbf{u}^{a}\right)^{2}
$$

$$
\frac{d \mathrm{u}^{a}}{d s}=\frac{d t}{c d \tau} \frac{d \mathrm{u}^{a}}{d t}=\frac{\sqrt{2} \mathrm{n}[\rho]}{c_{0}} \mathrm{a}^{a}
$$

where we have used (6.3) and (6.30), and the fact that:

from (4.7), and:

$$
\frac{d t}{d \tau}=\gamma=\sqrt{2}
$$

$$
c=\frac{c_{0}}{\mathrm{n}[\rho]}
$$

from (5.8), we may rewrite (7.2) in the following way:

$$
\mathrm{a}^{b}=\left[\frac{1}{2} \sum_{i=i}^{3} \ln \left[\mathrm{u}^{i}\right]-\frac{\left(1-\frac{\mathrm{R}_{\mathrm{S}}}{4 \rho}\right)^{2}}{\left(1+\frac{\mathrm{R}_{\mathrm{S}}}{4 \rho}\right)^{2}}\right]^{\frac{1}{2}} \frac{c_{0}}{\hbar}\left(1+\frac{\mathrm{R}_{\mathrm{S}}}{4 \rho}\right)^{-2} \hat{\mathbf{P}} \mathrm{u}^{b}
$$

where we have also used (5.6). Equation (7.6) represents the acceleration corresponding to spacetime translational component "b" in the Minkowski tangent space defined at $\mathrm{P}$, measured by an observer $O$ who remains stationary with respect to spacetime event $\mathrm{P}$, and resulting from the translation of a four-vector force along a closed curve in a Riemann-Cartan spacetime having zero curvature, but nonzero torsion. In other words, the components of $a^{b}$ correspond to the components of the vector cosmic accelerated expansion. From (2.2), (4.17) and (5.6) we now arrive at:

$$
\mathrm{F}^{a b}=\frac{\sqrt{2}\left(m_{O}\right)^{a}}{\hbar \lambda_{C}{ }^{-1} \sum_{i=1}^{3} d\left(\ln \left[\mathrm{u}^{i}\right]\right) d t}\left[\frac{1}{2} \sum_{i=i}^{3} \ln \left[\mathrm{u}^{i}\right]-\frac{\left(1-\frac{\mathrm{R}_{\mathrm{S}}}{4 \rho}\right)^{2}}{\left(1+\frac{\mathrm{R}_{\mathrm{S}}}{4 \rho}\right)^{2}}\right]^{\frac{1}{2}}\left(1+\frac{\mathrm{R}_{\mathrm{S}}}{4 \rho}\right)^{-2} \hat{\mathbf{P}} \mathrm{u}^{b}
$$

Equation (7.7) provides a geometrical description of the expression of force accompanying the action of the acceleration corresponding to spacetime translational component " $b$ " $(b=0,1,2,3)$ in the Minkowski tangent 
space defined at $\mathrm{P}$, on the proper inertial mass corresponding to spacetime translational component "a" $(\mathrm{a}=0,1$, $2,3)$ for an observer $O$ who remains stationary with respect to spacetime event $\mathrm{P}$, and resulting from the translation of a four-vector force along a closed curve in a Riemann-Cartan spacetime having zero curvature, but nonzero torsion. In other words, the tensor component $\mathrm{F}^{a b}$ expresses the action of the vector component $\mathrm{a}^{b}$ of the cosmic accelerated expansion on the geometric expression of proper inertial mass $\left(m_{O}\right)^{a}$ corresponding to the spacetime component "a" of an observer $O$, who is stationary with respect to spacetime event $\mathrm{P}$.

If spacetime is viewed as an elastic medium, a hypothesis for which the results obtained by the Gravity Probe B experiment[14] provide ample evidence, then the source of the components of the cosmic accelerated expansion is related to the areal density of a continuum version of the Burgers vector, where the Burgers vector, $d \mathbf{x}$, is a consequence of the translation of a four-vector force (given by (4.18)) along a closed curve in a Riemann-Cartan spacetime having zero curvature, but nonzero torsion. The Burgers vector corresponds to a measure of the distribution of dislocations in spacetime, where we identify a dislocation as any region contained within the Schwarzschild radius, $\mathrm{R}_{\mathrm{S}}$, of a black hole, which may, for example, be located at a galactic center.

\section{Conclusion}

In this paper, we develop the idea of mass as a geometric property of spacetime. Using this formalism, we present the analogue of Newton's second law for a spacetime having zero curvature, but nonzero torsion. In particular, we emphasize that if spacetime is described by a Riemann-Cartan manifold, the vanishing of curvature is not a sufficient condition for the exact validity of the results of the Special Theory of Relativity: one must also account for the presence of nonzero torsion, by taking the limit as the torsion approaches zero. When this is done, one is able to write down a Euclidean version of the Schwarzschild line element that is the (only spherical, by Birkhoff's theorem,) solution to the vacuum form of the Einstein field equations, in a manner which is suggestive of a connection to non-relativistic quantum mechanics. Finally, we have shown that the vector components of the cosmic accelerated expansion will correspond, in principle, to measurements associated with the translation of a point in the Minkowski tangent space which accompanies motion associated with a closed curve in a corresponding Weitzenböck manifold. This finding encourages the possibility that crystallographic models of elastic media with dislocations may allow us to use the formalism presented here to accurately determine the spacetime translational components in the Minkowski tangent space (defined with respect to observer $O$ at spacetime event $\mathrm{P}$ ) of the proper inertial mass associated with modeling spacetime as a Riemann-Cartan manifold. If it is found that these components are indeed anisotropic, resulting in an anisotropic vector accelerated expansion, such a discovery might serve as a decisive factor in enabling scientists to resolve current uncertainty surrounding why there are two prevailing measurements corresponding to the cosmic accelerated expansion.[15][16]

\section{Acknowledgements}

The author wishes to thank Kazuko Ota Cottrell for assistance throughout the writing of this paper.

\section{References}

[1] R. Ellis, P. Ferreira, R. Massey, and G. Weszkalnys, 90 years on - the 1919 eclipse expedition at Príncipe, Astronomy and Geophysics, 50, August 2009, 4.12-4.15.

[2] K. O. Cottrell and J.-P. Hsu, New perspectives on an old problem: the bending of light in Yang-Mills gravity, Proc. Twelfth AsiaPacific International Conference, Singapore, 2016, 378-381.

[3] K. O. Cottrell, New insights into the universe based on a unified approach, doctoral dissertation, University of Massachusetts Dartmouth, North Dartmouth, MA, 2015.

[4] K. Hayashi and T. Shirafuji, Gravity, nonintegrable phase factor and motion associated with a closed curve, Progress of Theoretical Physics, 58(1), 1977, 353-361.

[5] D. L. Bennett, C. R. Das, L. V. Laperashvili, and H. B. Nielsen, The relation between the model of a crystal with defects and Plebanski's theory of gravity, arXiv:1209.2155v2, [hep-th], October 20, 2012.

[6] H. F. M. Goenner, On the history of unified field theories, Living Rev. Relativity, 7(2), 2004, 1-152. [Online Article]: cited [January,21, 2017], http://www.livingreviews.org/lrr-2004-2 (In particular, see section 6.4 Distant parallelism).

[7] A. Ozakin and A. Yariv, Affine development of closed curves in Weitzenböck manifolds and the Burgers vector of dislocation mechanics, Mathematics and Mechanics of Solids, 19(3), (2012), 299-307.

[8] D. J. Griffiths, Introduction to electrodynamics, third edition (Upper Saddle River, NJ: Prentice-Hall, Inc., 1999). See equation (12.60) on page 516 .

[9] M. Moshinsky, On the interactions of Birkhoff's gravitational field with the electromagnetic and pair fields, Physical Review, 80(4), 1950, 514-519.

[10] P. Donis, The Schwarzschild geometry: part 1, Physics Forums Insights, December 12, 2016. [Online Article]: cited January 24, 2017], https://www.physicsforums.com/insights/schwarzschild-geometry-part-1/\#toggle-id-1

[11] R. Adler, M. Bazin, and M. Schiffer, Introduction to general relativity, second edition (New York, NY: McGraw Hill, 1975). See section 6.2, pages 196-199.

[12] F. J. Dyson, The radiation theories of Tomonoga, Schwinger, and Feynman, Physical Review, 75(3), 1949, 486-502.

[13] E. Merzbacher, Quantum Mechanics (New York, NY: John Wiley \& Sons, Inc., 1961). See page 42 and Table 4.1, page 43. 
[14] C. M. Will, Viewpoint: finally, results from Gravity Probe B, Physics, 4, May 31, 2011, 43-49. [Online Article]: cited [January 29, 2017], https://physics.aps.org/articles/v4/43

[15] C. Moskowitz, Cosmic speed measurement suggests dark energy mystery, Scientific American, April 11, 2016. [Online Article]: cited [January 26, 2017], https:/www.scientificamerican.com/article/cosmic-speed-measurement-suggests-dark-energy-mystery/

[16] M. Wall, Cosmic lenses show universe expanding surprisingly fast, Scientific American, January 26, 2017. [Online Article]: cited [January 28, 2017], https://www.scientificamerican.com/article/cosmic-lenses-show-universe-expanding-surprisinglyfast/?WT.mc id=SA DD 20170126

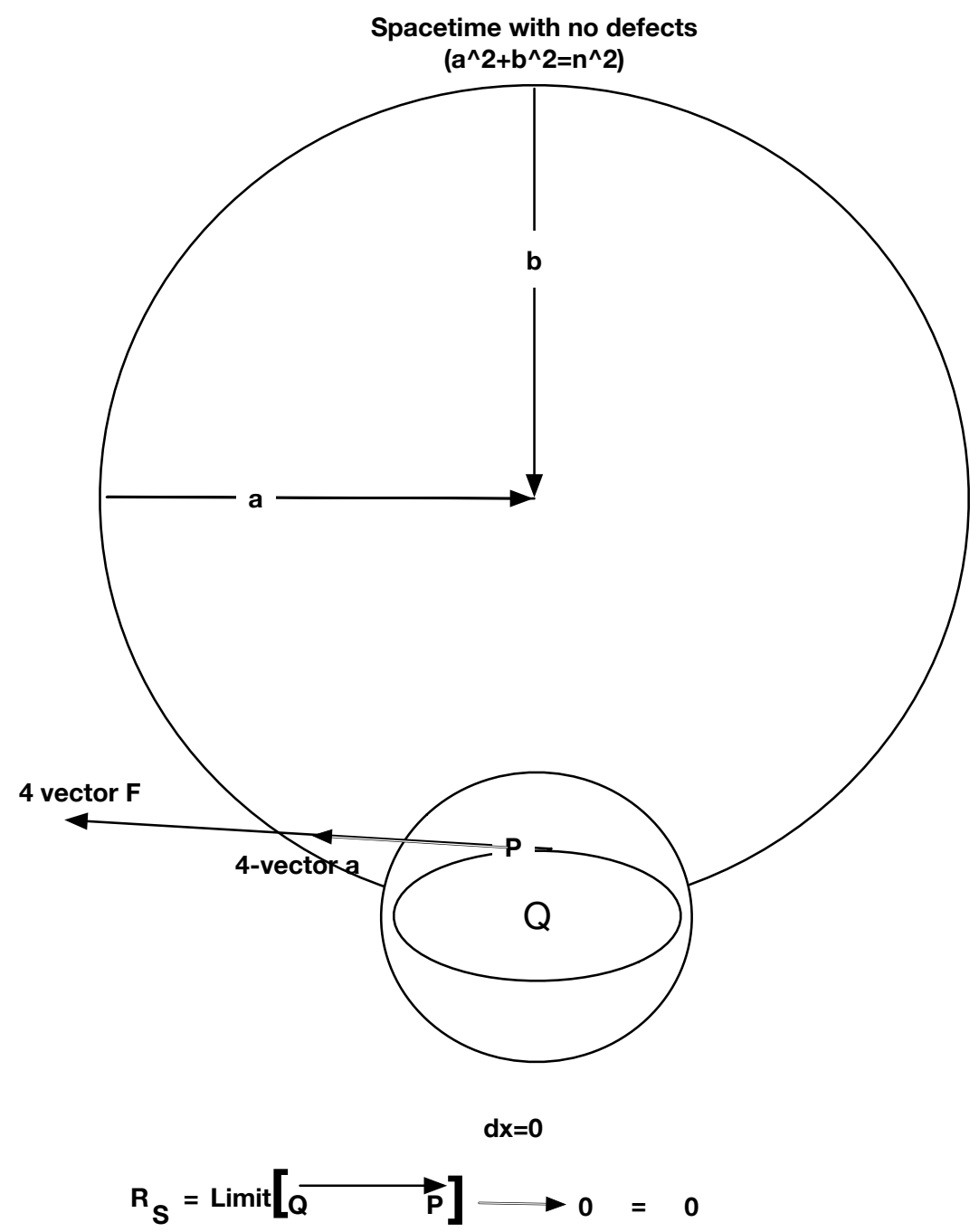

Figure 1. Diagram depicting motion of a four-vector force along a closed curve in a spacetime without defects. One can imagine the force to be acting at point $\mathrm{P}$, which is the spacetime location of observer $\mathrm{O}$, the proper observer for whom each unit vector of a coordinate basis corresponds also to a component of proper inertial mass $\left(m_{O}\right)^{a}$. One can imagine this point to be defined on the surface of a marble (size greatly exaggerated here) with origin at point $\mathrm{Q}$ (i.e., defined on the boundary of a neighborhood of $\mathrm{Q}$ ) in the absence of friction, so that the marble slips along the curve, or, equivalently, rolls along a circular curve of integral radius with no distortion, and the force vector returns with its tip at the original position (i.e. there is no rotation because the space has zero curvature, and there is no translation of the tip of the vector due to motion along the closed curve), so that effectively the length of segment QP is equal to zero. In such a space, there would be no cosmic accelerated expansion. 


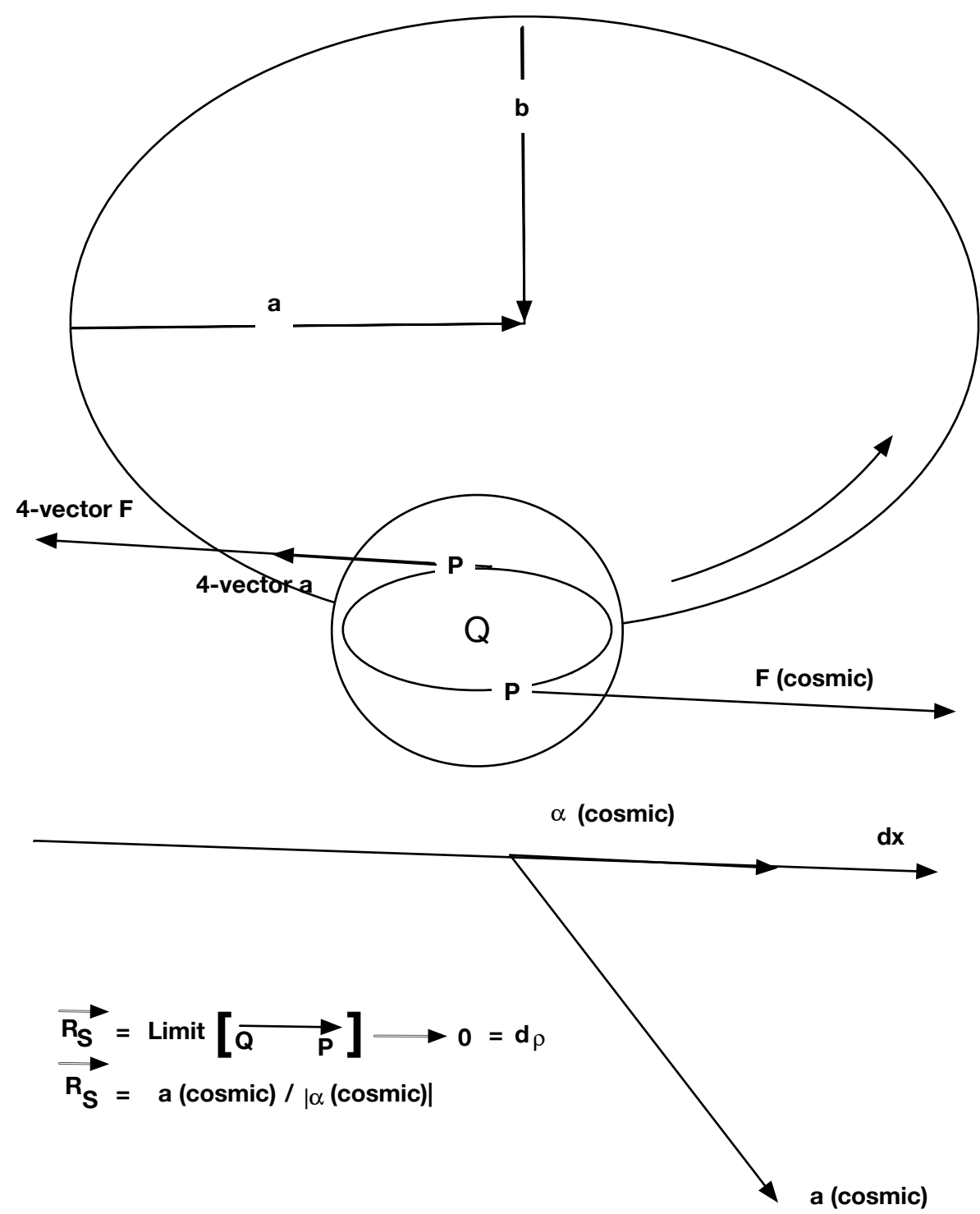

Figure 2. Diagram depicting motion of a four-vector force along a closed curve in a spacetime with defects. In a spacetime with defects, one can imagine that the marble is moving on a perfectly rough surface, so that it rolls without slipping. In this case, the marble rolls along the closed curve, which is now elongated (due to distortions resulting from the presence of defects), following an elliptical path, and the force vector returns with its tip antiparallel to the orientation of its original position: i.e., there is no net rotation of the force vector, because the spacetime has zero curvature, but there is a net translation $d \mathbf{x}$ of the tip of the force vector in the Minkowski tangent space due to motion of the force vector along the closed curve in a Weitzenböck spacetime with defects. The radius of the marble, segment QP (greatly exaggerated here) corresponds to the Schwarzschild radius of a black hole representing a defect with origin at Q. From the perspective of an observer at P, the mass $M$ of this black hole is a geometric property of spacetime, with components corresponding to each component of a set of basis vectors with origin at $\mathrm{P}$. Because of the net translation of the tip of the force vector in the Minkowski tangent space, there is an angular cosmic acceleration. The effective separation $1 / 2(2 \mathrm{QP})$ of the origins of an accelerated observer $O^{\prime}$ and a proper observer $\mathrm{O}$, respectively, corresponds to the Schwarzschild radius of a defect located at the origin of the coordinates $\mathrm{Q}$ for an arbitrary observer O', but at the origin of coordinates $\mathrm{P}$ for the proper observer $\mathrm{O}$ located at $\mathrm{P}$. The product of the magnitude of the angular cosmic acceleration with the Schwarzschild radius vector $\vec{R}_{S}$ yields the (possibly anisotropic) components of the cosmic acceleration vector. 